\title{
El sistema de producción de leche en Australia y Nueva Zelanda, y su reestructuración productiva
}

DOI: $10.32870 /$ mycp.v13i37.337

Ramón Robledo Padilla ${ }^{1}$

\section{Introducción}

$\mathrm{E}$ n este artículo se presenta un panorama general del sistema de producción lechera de Australia y Nueva Zelanda. Se analizan algunas de las razones que hacen posible que el sistema de producción de leche en estos países sea uno de los más competitivos dentro de los países desarrollados. También se aborda la importante participación de ambas naciones en el mercado internacional de lácteos, a pesar de que este sector se encuentra muy regulado por parte de la mayoría de los países desarrollados. Finalmente, se abordan las reformas estructurales que se han realizado en el sector lechero, las cuales forma parte de una estrategia general de liberación económica de ambos países conforme a los compromisos asumidos en el ámbito internacional.

\section{Sistemas de producción de leche}

\section{El caso de Australia}

Australia se caracteriza por ser uno de los países más competitivos en la producción de leche a escala mundial; esta característica se debe principalmente a sus condiciones naturales que le permiten operar con costos de producción muy bajos. Las condiciones naturales dan como resultado una calidad del pasto natural que se utiliza como principal alimento del ganado, el cual permite

1. Profesor investigador del Departamento de Estudios del Pacífico, del Centro Universitario de Ciencias Sociales y Humanidades, Universidad de Guadalajara. 
reducir los costos y aumentar la cantidad de leche producida. La eficiencia en el costo de producción es producto del desarrollo tecnológico, así como de las investigaciones llevadas a cabo para mejorar la producción de pastos y el mejoramiento genético. ${ }^{2}$

Australia - junto con Nueva Zelanda- es un caso excepcional cuyo sistema de producción de leche basado en el pastoreo le permite obtener los costos de producción más bajos

Australia - junto con

Nueva Zelanda- es un caso

excepcional cuyo sistema

de producción de leche

basado en el pastoreo le

permite obtener los costos de producción más bajos entre los países desarrollados. Su competitividad se basa, en mayor medida, en sus ventajas comparativas $y$, en menor medida, en la intervención de las políticas gubernamentales entre los países desarrollados. Su competitividad se basa, en mayor medida, en sus ventajas comparativas y, en menor medida, en la intervención de las políticas gubernamentales. Por lo tanto, podemos decir que la competitividad se percibe como el resultado combinado de los efectos de las distorsiones de mercado (provocadas tanto por políticas como por la competencia imperfecta) y las ventajas comparativas. $^{3}$

Dentro de este esquema general de producción basado en el pastoreo, existen dos subsistemas. Uno de ellos se utiliza en dos terceras partes de las granjas lecheras en Australia y se conoce como sistema estacional; en él se planea el apareamiento del ganado con anticipación, con el fin de que logren tener sus crías y dé comienzo el periodo de lactancia en la estación del año donde hay abundancia de pastos naturales debido a las lluvias, esto sucede normalmente durante la primavera, principalmente en los estados de Victoria y Tasmania. En ambos estados, a partir del mes de julio comienza a incrementarse la producción de leche hasta alcanza su nivel más alto en el mes de octubre, posteriormente la producción comienza a descender y llega al nivel más bajo en los meses de abril, mayo y junio.

2. Cfr. Australian Dairy Corporation, Annual Report, 1989-1990, p. 5.

3. Velázquez, Víctor Manuel, 1998, p. 81. 
El segundo subsistema de producción más importante en Australia es aquél que busca que el ganado tenga a sus crías durante todo el año, con el fin de mantener la producción de leche lo más estable posible. Este sistema se practica en las regiones donde es menor la variación estacional en la producción de pasturas, como en los estados de Nueva Gales del Sur, Queensland, el norte y el occidente de Australia. ${ }^{4}$

\section{El caso de Nueva Zelanda}

De manera similar aunque en mayor grado que Australia, Nueva Zelanda también basa su sistema de producción de leche en el pastoreo. Este sistema es poco común a escala internacional; en la actualidad, se calcula que aproximadamente sólo $10 \%$ del ganado lechero en el mundo utiliza como principal componente de alimentación el pasto; esta forma de explotación se encuentra en Nueva Zelanda, Australia y algunas zonas de Sudáfrica y Sudamérica. ${ }^{5}$ A diferencia de los sistemas de producción estabulados, donde el ganado permanece gran parte del tiempo en los establos y su dieta se compone de forraje y granos, las condiciones naturales de Nueva Zelanda, que incluyen suelos con una abundante capa de materia orgánica, distribución excelente de lluvias y un invierno benigno, permiten evitar la estabulación invernal y contar con pastos durante todo el año sin necesidad de riego. ${ }^{6}$ Esta forma de explotación necesariamente requiere un espacio amplio para el ganado, y es así como está organizado. Actualmente, en Nueva Zelanda existen aproximadamente 12,000 rebaños lecheros y, en promedio, cada uno cuenta con 315 bovinos en una extensión promedio de 115 hectáreas, es decir 2.7 cabezas de ganado por hectárea. ${ }^{7}$

Las características naturales mencionadas anteriormente, además del desarrollo de innovaciones tecnológicas, han permitido que la producción de leche en Nueva Zelanda se realice con el costo de producción más bajo dentro

4. Meter Martin and Phantipa Puangsumalee, Farm performance in the Australian and New Zealand dairy industries. Australian Bureau of Agricultural and Resource Economics. Disponible en: www.abareconomics.com

5. Disponible en: http://www.agritech.org.nz/dairy.shtml, p. 2.

6. Fideicomisos Instituidos en Relación con la Agricultura, FIRA. Boletín informativo, núm. 317, vol. xxxIII, Año 2001, p 38. Disponible en: http://www.siap.sagarpa.gob.mx.

7. Agricultural economies of Australia and New Zealand. Disponible en: http://www.abareconomics.com. 
de los países desarrollados; sin embargo, es precisamente el sistema de producción basado en el pastoreo con bajo uso de granos, lo que también influye en los rendimientos inferiores en la producción de leche: hasta $50 \%$ con respecto a las granjas con manejo estabulado y uso de alimentos concentrados. ${ }^{8}$

\section{Cuadro 1}

Promedio anual de producción de leche por vaca (litros)

\begin{tabular}{llll} 
País & 2000 & 2003 & 2007 \\
\hline Nueva Zelanda & 3,666 & 3,652 & 3,817 \\
Australia & 5,151 & 4,914 & 5,132 \\
Alemania & 6,076 & 6,489 & 6,807 \\
Estados Unidos & 8,036 & 8,262 & 8,931 \\
Canadá & 7,396 & 7,259 & 7,730 \\
México & 3,296 & 3,240 & 3,855 \\
\hline
\end{tabular}

Fuente: www.fao.org.

Como se puede observar en el cuadro anterior, mientras en Nueva Zelanda cada bovino produce poco más de 3,600 litros de leche en promedio anual, en los Estados Unidos la producción promedio en esos mismos años fue de más del doble, en Alemania cercana al doble y en Canadá prácticamente del doble; mientras tanto, en Australia el rendimiento en la producción de leche fue aproximadamente de $35 \%$ más del que se registró en Nueva Zelanda. Aunque los sistemas de producción entre Nueva Zelanda y Australia son parecidos, uno de los aspectos importantes que los distinguen es el hecho de que en Nueva Zelanda su sistema se basa en mayor medida en el pastoreo, con mucho menos uso de suplementos alimenticios que el sistema de producción australiano. ${ }^{9}$

\section{Participación en el mercado internacional}

\section{El caso de Nueva Zelanda}

La razón por la que analizo en primer lugar a Nueva Zelanda en lugar de Australia - que era el orden en el que lo venía haciendo- se debe al hecho de que Nueva Zelanda tiene una mayor relevancia en el mercado internacional.

8. Op. cit. Fideicomisos Instituidos en Relación con la Agricultura, FIRA, p. 40.

9. Op. cit., Meter Martin. 
Comúnmente, la mayoría de los países consumen casi el total de la leche que producen, y sólo una parte marginal se destina al comercio internacional. Hoy en día, poco más de $15 \%$ de la producción mundial (alrededor de 80 mil millones de litros) es lo que se comercia internacionalmente, incluyendo lo que se comercia entre los países de la Unión Europea; pero, si se excluye el comercio entre estos países, el comercio mundial de lácteos en el resto del mundo sería aproximadamente de $10 \%$ de la producción mundial total.

\section{Cuadro 2}

Producción mundial de leche (millones de litros)

\begin{tabular}{lrrrrrr}
\hline País & \multicolumn{1}{c}{2000} & \multicolumn{1}{c}{2003} & \multicolumn{1}{c}{2004} & \multicolumn{1}{c}{2005} & \multicolumn{1}{c}{2006} & \multicolumn{1}{c}{$2007^{*}$} \\
\hline Nueva Zelanda & 12,235 & 14,349 & 15,030 & 14,637 & 15,332 & 15,814 \\
Australia & 11,183 & 10,075 & 10,125 & 10,089 & 10,250 & 10,350 \\
Unión Europea & 155,462 & 155,326 & 153,193 & 154,120 & 153,372 & 151,733 \\
Estados Unidos & 76,023 & 77,289 & 77,535 & 80,254 & 82,463 & 84,189 \\
México & 9,311 & 9,784 & 9,864 & 9,868 & 10,088 & 9,599 \\
Mundo & 490,530 & 517,073 & 525,543 & 541,342 & 555,704 & 527,667 \\
\hline
\end{tabular}

*Preliminar.

Fuente: http://www.fao.org.

Cuadro 3

Exportación mundial de lácteos (equivalente en millones de litros)

\begin{tabular}{lrrrrrrrr}
\hline País & \multicolumn{1}{c}{ 2000 } & \multicolumn{1}{c}{$\%$} & \multicolumn{1}{c}{2003} & \multicolumn{1}{c}{$\%$} & \multicolumn{1}{c}{2004} & \multicolumn{1}{c}{$\%$} & \multicolumn{1}{c}{2005} & \multicolumn{1}{c}{$\%$} \\
\hline Nueva Zelanda & 8,657 & 11.88 & 11,357 & 14.51 & 10,795 & 12.80 & 10,147 & 11.76 \\
Australia & 5,579 & 7.65 & 4,540 & 5.80 & 4,925 & 5.84 & 4,664 & 5.40 \\
Unión Europea & 48,166 & 66.07 & 50,164 & 64.11 & 52,647 & 62.41 & 53,250 & 61.70 \\
Estados Unidos & 2,885 & 3.96 & 2,826 & 3.61 & 4,143 & 4.91 & 4,715 & 5.46 \\
Mundo & 72,899 & 100.00 & 78,251 & 100.00 & 84,360 & 100.00 & 86,306 & 100.00 \\
\hline
\end{tabular}

Fuente: http://www.fao.org.

De acuerdo con estas cifras de la FAO — no actualizadas-, Nueva Zelanda exporta una cantidad de lácteos equivalente en leche a $70 \%$ de su producción interna; sin embargo, de acuerdo con datos de la Secretaría de Agricultura y Silvicultura del gobierno de Nueva Zelanda, sus exportaciones superan $90 \%$ de su producción interna. De cualquier manera, la cifra es muy considerable y 
se debe a que se ha desarrollado una industria lechera muy competitiva frente a un mercado interno que es relativamente pequeño, lo que la convierte en el mayor exportador de lácteos del mundo. Si se analizan las exportaciones mundiales de leche, incluyendo el comercio que se da entre países de la Unión Europea, a esta región correspondería $60 \%$ de las exportaciones totales, a Nueva Zelanda poco más de $10 \%$ y en tercer lugar estaría Australia con casi $6 \%$ de participación. Dado que una gran parte de este comercio se realiza entre países de la Unión Europea — que equivale a 35 mil millones de litros, aproximadamente-, lo anterior quiere decir que, si excluyéramos este comercio, la participación de la Unión Europea en el comercio internacional de lácteos con el resto del mundo se reduciría a sólo 35\%, la de Nueva Zelanda se incrementaría a $20 \%$ y la de Australia también se incrementaría a $10 \%$; es decir, Nueva Zelanda por sí sola estaría contribuyendo con una quinta parte del total de exportaciones lácteas en el mundo. Aunque en los últimos años Estados Unidos exporta una cantidad equivalente de leche similar a la de Australia, en términos monetarios Australia continúa ocupando el tercer lugar, debido a que sus exportaciones lácteas son de un mayor valor agregado que las de Estados Unidos, por ejemplo, en 2005 las exportaciones de Estados Unidos alcanzaron un valor de 1,123 millones de dólares y las de Australia 1,860 millones de dólares estadounidenses. ${ }^{10}$

\section{Principales mercados de exportación}

De acuerdo a información del Ministerio de Agricultura y Silvicultura del gobierno de Nueva Zelanda, el principal mercado de exportación (39\%) es la región del sudeste de Asia, le siguieron Canadá y Estados Unidos con 14\%, el resto de América con 10\%, la Unión Europea con 8\%, y finalmente el resto de los países con 29\%. La participación que tiene Asia como principal destino de las exportaciones lácteas de Nueva Zelanda ha sido propiciada por una serie de factores como el creciente nivel de ingresos de la población en la región y los cambios en los patrones de consumo, así como por la búsqueda de diversificación de mercados que emprendió el gobierno de Nueva Zelanda a partir de 1972, año en que el Reino Unido, principal mercado de exportación, decidiera formar parte de la hoy conocida Unión Europea y con ello se vieran disminuidas las ventajas que este mercado proporcionaba. Ante tal situación,

10. http://www.fao.org. 
a finales de los años sesenta, a Nueva Zelanda no le quedó otro camino que buscar nuevos mercados de consumo, ya que sus exportaciones de lácteos hacia el reino Unido concentraban $90 \%$ de su producción. ${ }^{11}$

\section{Gráfica 1}

Nueva Zelanda: exportación de lácteos por región, 2006-2007

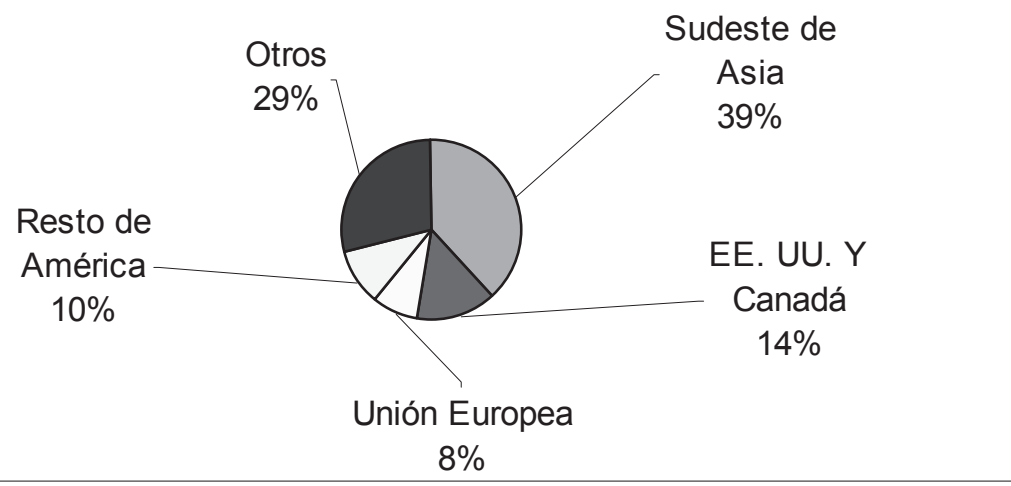

Fuente: http://www.maf.govt.nz.

El caso de Australia

En forma similar al caso de Nueva Zelanda, aunque en menor proporción, también en Australia la producción interna de leche supera la demanda interna. De este modo, Australia exporta cerca de $50 \%$ de la producción interna.

También de manera similar al caso de Nueva Zelanda, Australia concentra la mayor parte de sus exportaciones en el mercado de Asia, con Japón como su principal mercado, donde concentra $20 \%$ de sus exportaciones totales. Australia, al igual que Nueva Zelanda, también tuvo graves problemas en su industria láctea a inicios de los años setenta, en primer lugar porque en 1973 Gran Bretaña decidió formar parte de la Unión Europea y con ello terminaron las tarifas preferenciales para Australia, en segundo lugar, hubo una fuerte campaña en Australia y en los principales mercados de Europa orientada al consumo de productos más sanos - como el de la margarina en lugar de la mantequilla- lo que provocó que en 1972-1973 el mercado de la mantequilla, tanto interno como externo, disminuyera $24 \%$ y $41 \%$ más al año siguiente;

11. http://europa.eu.int/. 


\section{Gráfica 2}

Australia: principales destinos de exportación, 2006-2007

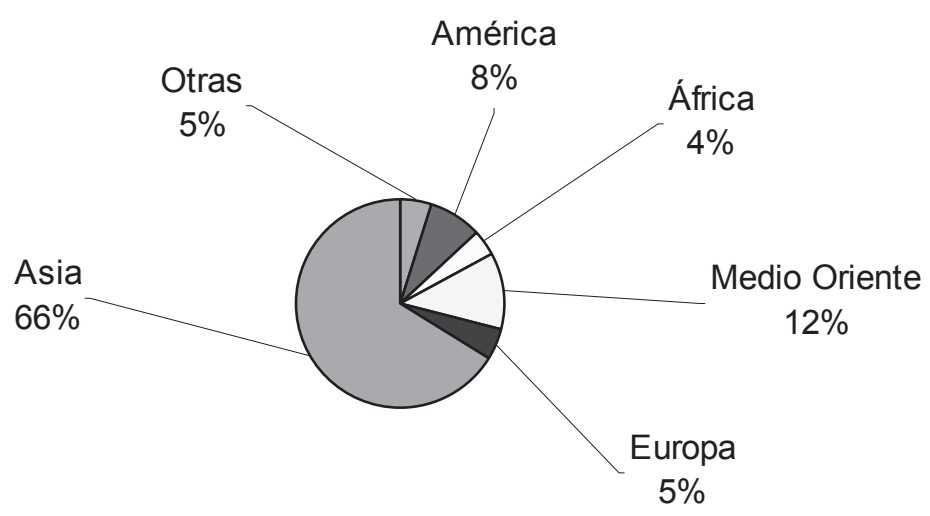

Fuente: http://wwww.dairyaustralia.com.au.

finalmente, como resultado de los excedentes lácteos acumulados en Europa y los Estados Unidos, los precios internacionales de la leche disminuyeron. Lo anterior provocó que en Australia se diera una reducción en la producción de leche, una diversificación en la producción de lácteos y una búsqueda de nuevos mercados. Finalmente, a partir de los años ochenta, la industria se fue recuperando poco a poco, una vez que la producción mundial de leche había disminuido como consecuencia de una reducción de los subsidios y de otros apoyos que se aplicaban principalmente en países importantes de la Unión Europea y los Estados Unidos. ${ }^{12}$

\section{Apoyos al sector lácteo}

En la mayoría de los países desarrollados, con excepción de Australia y Nueva Zelanda, existe una gran variedad de mecanismos de apoyos a la producción agropecuaria (incluyendo los de la leche). Tal es el caso de algunas de las economías más avanzadas como las de Europa y los Estados Unidos. Los apoyos son muy diversos, comprenden desde subsidios a la exportación y a los

12. Australian Dairy Industry Council, The comprehensive reference to the Australian Dairy Industry, Morescope Publishing Pty Ltd., 1996, pp. 17 y 18. 
diferentes insumos para la producción, hasta restricción a las importaciones y fijación de precios que garantizan la obtención de un beneficio positivo, etcétera. Australia y Nueva Zelanda son países desarrollados que mantienen en la actualidad una política de apoyos muy bajos hacia el sector agrícola. El país que en menor proporción apoya al sector agropecuario es Nueva Zelanda. Sin embargo, Australia es el país que más se acerca en cuanto al comportamiento de sus políticas agrícolas. Así lo muestran las reformas que ha puesto en marcha en los últimos años el gobierno australiano, el cual se orienta hacia una política de menor intervención en el sector agropecuario. ${ }^{13}$

Los subsidios agrícolas, otorgados principalmente por países desarrollados, provocan incrementos a la producción que finalmente se traducen en una baja en los precios a escala internacional, esto hace más difícil que los países pobres puedan competir con sus productos primarios en un mercado altamente subsidiado. Debido a la baja en los precios internacionales de algunos productos primarios, muchos países subdesarrollados optan por incrementar sus importaciones de estos productos, lo cual provoca serios daños a su sector agropecuario interno. Para los casos de Nueva Zelanda y Australia, que son países desarrollados, ${ }^{14}$ pero muy eficientes en la producción de leche con fuerte presencia en los mercados internacionales, la baja en los precios de la leche provocada por los elevados subsidios que se destinan a la producción en otros países tiene una repercusión directa en el sector lácteo de estos países, debido a que se reducen sus niveles de ingreso.

Entre los países desarrollados, Nueva Zelanda es el que otorga menos subsidios a su sector lácteo. Una forma de medir estos apoyos es a través de un indicador conocido como la Estimación de la Ayuda al Productor ${ }^{15}$ (PSE por sus siglas en inglés), antes conocido como el Equivalente del Subsidio al Productor; este indicador es una medida adoptada por la OCDE para medir el grado de ayuda a la agricultura. Actualmente, el PSE continúa calculándose para la agricultura en general; sin embargo, el PSE por producto ya no se calcula y la última vez que apareció en los reportes de la OECD fue en 2005. A partir de ese año, el indicador que mide las transferencias por producto es la Single

13. Cfr. 105 World Bank Discussion, p. 8.

14. Esto de acuerdo a consideraciones de la ONU, que establece que son países desarrollados aquéllos que cuentan con un índice de desarrollo humano (IDH) de entre .800 y 1 , y un ingreso per cápita de 18,500 dólares en adelante.

15. La Estimación de Ayuda al Productor es un indicador del valor de las transferencias monetarias a la agricultura que resultan de las políticas agrícolas en un año dado. 
Commodity Transfers (SCT), o transferencias destinadas a un bien. Éste, a diferencia del PSE que se calculaba por producto, mide los apoyos que están más directamente relacionados con ese bien, por ejemplo, los apoyos que se reciben por incrementar la producción; en cambio, el PSE por producto incluía algunos apoyo implícitos como son la reducción de los costos de producción debidos por ejemplo a reducción de impuestos, o también el acceso al crédito, energía y agua a un costo menor que el resto de la sociedad.

\section{Cuadro 4}

Transferencias monetarias a los productores de leche, en porcentaje

\begin{tabular}{lcrrr}
\hline País & $2005-2007 \%$ & $2005 \%$ & $2006 \%$ & $2007 \%$ \\
\hline Nueva Zelanda & 0 & 0 & 0 & 0 \\
Australia & 22.0 & 67 & 0 & 0 \\
Canadá & 48.4 & 48.9 & 58.8 & 37.4 \\
Estados Unidos & 19.1 & 18 & 16.6 & 22.7 \\
Unión Europea (15) & 14.3 & 23.3 & 19.6 & nd \\
Japón & 50.6 & 58 & 54.2 & 39.5 \\
México & 5.4 & 1.2 & 15 & 0 \\
\hline
\end{tabular}

Fuente: Agricultural policies in OECD Countries, at a glance 2008, preliminary version, OECD, 2008.

De acuerdo con este nuevo indicador, como podemos apreciar en el cuadro 4, en Nueva Zelanda y Australia no se recibieron apoyos en los últimos dos años; en cambio, en Canadá y Japón, casi 50\% de los ingresos recibidos por los productores de leche provienen de subsidios; en los Estados Unidos y la Unión Europea alrededor de $18 \%$ de los ingresos del productor provienen de estos apoyos, y en México alrededor de 5\%. La conclusión de esto es que en los países desarrollados los subsidios destinados a la producción de leche continúan siendo elevados, a excepción de Australia y Nueva Zelanda.

Otro indicador que utiliza la OCDE para evaluar la ayuda otorgada a productores agropecuarios se conoce como el Coeficiente de Protección Nominal (NPC, por sus siglas en inglés), es un coeficiente muy simple el cual se emplea para evaluar el nivel de protección resultante de distorsiones de mercado. Lo que se mide es la relación entre el precio interno que reciben los productores por su producto y el precio en el mercado internacional de ese producto. En México, por ejemplo, para el caso de la leche este indicador fue de 1.18 en 
2006, esto quiere decir que en ese año el precio promedio del litro de leche en México fue $18 \%$ más alto que en el mercado internacional. En los Estados Unidos el indicador fue 1.20, en la Unión Europea 1.25, Canadá 2.43, Japón 2.18 y finalmente en Nueva Zelanda y Australia el NPC fue 1, lo cual indica que en estos países no hubo ayuda implícita, o que el precio que recibieron los productores de leche fue igual al precio en el mercado internacional.

\section{Reforma estructural}

\section{El caso de Australia}

Australia, al igual que la mayoría de los países, ha realizado cambios en su política económica interna en las últimas dos décadas. Estos cambios están orientados a reducir las intervenciones del gobierno y permitir que las actividades económicas se orienten únicamente por las señales del mercado.

De acuerdo con esta nueva realidad, en los años noventa Australia promovió un paquete de políticas orientadas a incrementar los beneficios de los trabajadores del campo y mejorar la competitividad en el ámbito internacional. ${ }^{16}$ En general, los apoyos proporcionados al sector rural antes de 1982 no fueron uniformes. Los más altos niveles de protección estuvieron dirigidos a la irrigación de cosechas, el sector lácteo, el de azúcar y el de huevo, mientras que otras actividades que eran consideradas más eficientes, como las de la lana y la carne de vaca, se mantuvieron sin intervención o con un nivel de apoyo relativamente bajo. ${ }^{17}$

La transición que se dio en Australia entre 1982 y 1992 estuvo orientada a responder a las señales del mercado y a la eliminación de las intervenciones del gobierno en forma gradual. Se lograron acuerdos entre el gobierno, la industria y el sector laboral para establecer salarios por abajo de la inflación con el fin de reducir los costos del trabajo y estimular la inversión; al mismo tiempo, el gobierno promovió la desregulación de los mercados financieros con el fin de que estos recursos estuvieran disponibles con mayor rapidez para los individuos y sus negocios. ${ }^{18}$

16. Disponible en: http://www.dpie.gov.au. Fecha de consulta: septiembre de 1997, p. 3.

17. Ibídem, pp. 6 y 7 .

18. Ídem, p. 7. 
Con esta serie de reformas, la agricultura fue tratada como parte integral de la economía sin ninguna consideración especial. Las medidas se orientaron a reducir los niveles de protección comerciales sobre las importaciones, con el fin de ir reduciendo en forma gradual las barreras al comercio.

En 1995 una gran cantidad de productos quedó libre de restricción; únicamente permanecieron con protección algunas industrias agrícolas como las de los cítricos, la leche, el azúcar, las frutas y otras en las que la reducción en la protección ha sido en forma gradual. ${ }^{19} \mathrm{El}$ apoyo al sector lácteo y la desregulación paulatina se debió a que, normalmente, este sector ha sido uno de los más protegidos en muchos de los países desarrollados, lo cual provocaba y continúa provocando una competencia no equitativa.

\section{Reforma en la política de la industria de la leche}

Hasta principios de los años ochenta, en Australia existía un control centralizado en el mercado de las exportaciones. Además, debido a que la industria de la leche no era muy grande en términos relativos y estaba formada por pequeñas cooperativas, la obtención de información sobre los mercados de exportación era muy costosa.

Dado lo anterior, el sector procesador fue regulado conjuntamente a través de acuerdos de exportación entre las pequeñas cooperativas productoras de leche con precios asegurados; además de esto, el precio interno en Australia era controlado por la legislación de cada uno de los estados.

Antes del primero de julio de 2000, la regulación en la industria de la leche de Australia se dividía en dos partes: por un lado, estaba el sector de leche líquida conocido como mercado de la leche (Market Milk) y por el otro, el sector de leche industrializada (Manufacturing Milk). Ambos sectores fueron apoyados de manera distinta. El mercado de leche líquida fue apoyado de manera independiente en cada uno de los estados de Australia, y el sector de leche industrializada consistió en un esquema de asistencia federal por la Commonwealth.

Los controles de los gobiernos estatales sobre el precio y la oferta de la leche líquida - conocida como mercado de leche- habían estado presentes por lo menos desde 1980 en la mayoría de los Estados, y en otros desde algunos años más. El precio que el Estado garantizaba por la producción de cierta

19. Ibídem, p. 9-10. 
cantidad de leche líquida, estaba muy por encima del precio promedio que se pagaba por el resto de la oferta de leche no regulada, ${ }^{20}$ a pesar de que no existían diferencias en el producto. De acuerdo con la política de desregular el mercado, las cuotas y los precios de control estatal finalizaron el primero de julio de 2000, esto significa que ya no existen controles sobre la cantidad o el precio de la leche producida, y por lo tanto existe un mercado libre en las ventas de leche líquida en Australia. ${ }^{21}$

En lo que concierne al apoyo por parte del gobierno de la Commonwealth, dos eran los programas más importantes que ofrecían asistencia al sector. Los programas se conocieron como el Plan Kerin y el Plan Crean de 1986 y 1992, respectivamente.

La estrategia del Plan Kerin, de 1986, se basaba en una política que mantenía los precios internos de los productos lácteos por arriba de los precios de exportación (aproximadamente dos centavos por litro), esto estaba ligado a un pago que se efectuaba a aquellas exportaciones de productos lácteos; a su vez, el dinero utilizado para estos fines provenía de un impuesto que se aplicaba a toda la producción de leche en Australia. Esta estrategia, que estimulaba el incremento de los precios internos de los lácteos, finalmente se traducía en un beneficio neto para los productores de leche, ya que se incrementaba el precio de la leche que era utilizada para la elaboración de productos lácteos destinados al consumo interno. ${ }^{22}$ El Plan Crean de 1992 sustituyó al anterior, y lo que planteó básicamente fue ir reduciendo los apoyos gradualmente hasta eliminarlos en junio 30 de 2000.

A partir de 1995, los apoyos tuvieron que ser rediseñados debido a que, de acuerdo a la OMC, el esquema de pagos para apoyar la exportación de productos lácteos constituía un subsidio a las exportaciones.

El nuevo esquema fue administrado por la Corporación Australiana de Leche (Australian Dairy Corporation, ADC), asimismo, las medidas fueron derivadas de los planes Kerin y Creen que — como se mencionó- fueron introducidos en 1986 y 1992, respectivamente. A partir de julio de 1995 se estableció esta nueva estrategia política con el propósito de cumplir con los

20. En la década de los noventa, el precio más bajo de la leche fluida controlada fue de 48 centavos australianos y el más alto de 55 centavos. En el mismo periodo, el precio promedio de la leche no controlada fue de entre 20 y 28 centavos en promedio.

21. Disponible en: http://www.aph.gov.au/library/pubs/bd/2000-01/01bd167.htm

22. Disponible en: http:www.dairy.com.au./adc/profile/markarr, p. 1. Fecha de consulta: mayo de 1997. 
acuerdos que Australia había contraído en el marco de la Ronda de Uruguay. Para esto, el nuevo plan trató de que los industriales de la leche mantuvieran los mismos beneficios que con el plan anterior. El cambio fundamental con respecto a los planes anteriores, consistía básicamente en que el apoyo interno se realizara de tal manera que no se interpretara como un subsidio a las exportaciones. Finalmente, la modificación quedó de la siguiente manera: los productores de leche pagaban un impuesto por la leche que vendían internamente para consumo directo (alrededor de dos centavos por litro), a su vez, los industriales de la leche pagaban un impuesto (en 1996-1997 el pago fue de alrededor de cuatro centavos por litro) por los productos lácteos que se vendían internamente. La leche utilizada para la elaboración de lácteos destinados a la exportación quedó exenta de impuestos. En este sentido, los apoyos a las exportaciones bajo esta lógica terminaron en junio 30 de 2000. Finalmente, los recursos de los impuestos que habían sido obtenidos mediante estas nuevas medidas, fueron utilizados para realizar pagos a aquellos productores cuya leche era destinada a la elaboración de productos lácteos, ${ }^{23}$ y los criterios para realizar dichos pagos se establecieron en función de la calidad y la cantidad de leche ofrecida.

De 1986 a 1996, la ayuda a la industria de leche había declinado aproximadamente en $65 \%$ y se esperaba fuera eliminada completamente, en forma gradual para el año 2000. No obstante la reducción de los apoyos por parte del gobierno desde 1986, una década después la producción de leche se había incrementado en una tercera parte y el volumen de las exportaciones habían aumentado al doble; lo anterior, a pesar de que hubo una reducción de $10 \%$ en el número de cabezas de ganado y una disminución de 30\% de las granjas lecheras. ${ }^{24}$

Para aminorar los efectos de la desregulación en el sector de la industria de leche, el gobierno de la Commonwealth introdujo una nueva legislación que comenzó a aplicarse el primero de julio del año 2000. A esta nueva ley se le conoce como Paquete de Ajustes a la Industria de la Leche (Dairy Industry Adjustment Package, DIAP), que comprende tres programas. ${ }^{25}$ El primero consiste en financiar, mediante pagos trimestrales durante los próximos ocho años

23. Ibídem, p. 2. Disponible en: http://www.dairy.com.au/adc/profile/dairystr, p. 2. Fecha de consulta: mayo de 1997.

24. Disponible en: http://www.dpie.gov.au/dpie/agriculture/agricultural_reform.html, p 12. Fecha de consulta: mayo de 1997.

25. ABARE, pp. 4-5. 
a partir de 2000, a todos aquellos granjeros que estuvieran interesados en continuar con la producción de leche. Para realizar estos pagos, se tomaron como referencia los volúmenes de producción de leche líquida e industrializada durante los años de 1998 a 1999.

El segundo programa consistió en apoyar con 45,000 dólares australianos (equivalentes a 25,875 dólares estadounidenses) libres de impuestos, a aquellos granjeros que desearan abandonar la actividad. Finalmente, el tercer programa tuvo como finalidad destinar recursos a las comunidades dependientes del sector lechero que fueron afectadas por los programas de desregulación.

\section{El caso de Nueva Zelanda}

Después de la II Guerra Mundial, Nueva Zelanda adoptó una fuerte política proteccionista, la cual se intensificó a finales de los años setenta y principios de los ochenta en un intento por conservar la prosperidad de los años cincuenta. ${ }^{26}$ Sin embargo, a partir de 1984, al igual que en muchos otros países en la década de los ochenta, esta nación comenzó un programa importante de liberalización económica. Los cambios en la economía de Nueva Zelanda durante los años ochenta se caracterizaron por su amplitud, la consistencia de las reformas y el bajo nivel de liberalización por el que comenzaron. $\mathrm{Mu}$ chos programas de la agricultura cambiaron dramáticamente: los esquemas de apoyo al precio fueron eliminados, se terminaron los préstamos para el desarrollo de las granjas y el subsidio a fertilizantes, además, la función del Banco Rural fue reorientada poco a poco hacia la comercialización de sus actividades, ello provocó el incremento en las tasas de interés, que aunado a la caída del ingreso agrícola, condujo a una crisis de endeudamiento rural. ${ }^{27}$

\section{Reforma en la política de la industria de la leche}

La liberalización en la economía de Nueva Zelanda implicó cambios en el sector agropecuario en general y en el sector lácteo en particular. Estos cambios

26. Evans Lewis. Structural reform: the dairy industry in New Zealand. APEC high level conference on structural reform, del 8 al 9 de septiembre de 2004, Tokio, Japón, p. 4.

27. www.ifama.org/ (Changes in risk perception and risk management strategies in New Zealand dairy farming), p. 4. 
propiciaron una mayor concentración de la producción de leche; por ejemplo, a mediados de los años ochenta el número de compañías lecheras (principalmente cooperativas) que existían en Nueva Zelanda eran 30, mientras que para 1998 quedaban solamente 8 . Este proceso condujo finalmente a una de las fusiones más grandes en 2001, en la que las dos principales cooperativas lecheras de Nueva Zelanda (New Zealand Dairy Group y Kiwi Dairies) y la Compañía Lechera de Nueva Zelanda, (The New Zealand Dairy Board, NZDB), acordaron formar una cooperativa conocida formalmente como Compañía Lechera Global (Global Dairy Company) ahora conocida como Grupo Cooperativo Fonterra (Fonterra Cooperative Group). Fonterra actualmente es la cooperativa más grande de Nueva Zelanda: recibe y procesa alrededor de 95\% de la leche producida en el país.

El antecedente más lejano de Fonterra inicia en 1923 con la creación del Consejo para el Control de la Exportación de Productos Lácteos (The Dairy Produce Export Control Board), el cual fue creado a iniciativa del gobierno con el fin de controlar todas las exportaciones de lácteos bajo el argumento de que las compañías procesadoras eran demasiado pequeñas para que por su propia cuenta pudieran exportar eficientemente. Posteriormente, este consejo sería conocido como The New Zealand Dairy Board (NZDB), la cual adquirió en 1961 legalmente el estatus de exportador único. Dicho estatus creaba serios problemas de libre competencia, debido a que, como el mercado de Nueva Zelanda era y continúa siendo demasiado pequeño, cualquiera que se iniciara en este negocio tendría que exportar sus productos con el fin de ampliarse; sin embargo, para hacerlo se debía solicitar autorización a sus competidores, los cuales eran propietarios de la compañía exportadora y, por lo tanto, implicaba una clara desventaja en términos de competencia. Entre otros factores, éste era uno de los puntos más cuestionados de la política gubernamental por lo que finalmente se logró impulsar un cambio estructural con el fin de eliminar esta condición de exportador único. ${ }^{28}$ Con la Ley de Reestructuración de la Industria de la Leche en 2001, el estatus de exportador único que mantenía la NZDB quedó eliminado, asimismo, se autorizó la fusión entre la NZDB y las dos cooperativas más grandes del país para dar origen a Fonterra; con ello, la responsabilidad de comercializar los lácteos en el mercado internacional, que otrora tenía la NZDB, pasa — al menos de manera temporal— a ser una responsabilidad de Fonterra.

28. Op. cit., Evans Lewis, p. 8. 
Con la reestructuración y la creación del Fonterra se logró poner fin al monopolio de exportación de productos lácteos. Mediante esta ley, aunque la empresa conserva su función de principal exportador debido a su experiencia y al volumen de leche que acopia y procesa, legalmente perdió su condición monopólica. Sin embargo, de acuerdo a la ley, la empresa mantendrá por el momento licencias exclusivas de exportación a determinados mercados restringidos, ${ }^{29}$ que estarán vigentes en algunos casos hasta 2007, y en otros hasta $2010 .^{30}$

Finalmente, de acuerdo a datos de la propia empresa, Fonterra es actualmente la compañía más grande de Nueva Zelanda; es propiedad — bajo la modalidad de cooperativa- de más de 12 mil ganaderos, sus exportaciones representan más de $20 \%$ del total realizadas por el país y participa con $7 \%$ del Producto Interno Bruto. Es además la empresa privada de Nueva Zelanda que más invierte en investigación y desarrollo, con un gasto anual aproximado de 95 millones de dólares neocelandeses, además de 3 millones adicionales que recibe del gobierno vía la Fundación para la Investigación, la Ciencia y la Tecnología (The Foundation for Research, Science and Technology). ${ }^{31}$

\section{Conclusión}

Los procesos de apertura comercial y desregulación económica que se han dado en Australia y Nueva Zelanda, y en particular en el sector lácteo, no han mermado en el largo plazo la cantidad de leche producida, la importante presencia de ambos países en el mercado internacional de lácteos, ni el incremento en su productividad. Lo anterior se debe a varias razones. En primer lugar, ambas economías han aprovechando sus condiciones naturales y han desarrollado, a lo largo de su historia, un sistema de explotación lechera basado en el pastoreo, el cual resulta muy eficiente en términos de costos de producción; en segundo lugar, se han llevado a cabo una serie de políticas

29. Disponible en: http://www.wto.org/english/tratop_e/tpr_e/s115-4_e.doc

30. Fonterra se beneficiará de licencias exclusivas de exportación hasta el 30 de junio de 2007 para las exportaciones al mercado de la República Dominicana, hasta el 31 de julio de 2007 para las exportaciones a determinados mercados del Canadá, hasta el 31 de diciembre de 2007 para las exportaciones a determinados mercados de la Comunidad Europea, hasta el 31 de diciembre de 2008 y 31 de diciembre de 2009 para las exportaciones de determinados quesos al mercado de los Estados Unidos, y finalmente, tendrá licencia exclusiva hasta el 31 de marzo de 2010 para las exportaciones al mercado japonés.

31. Disponible en: http:www.fonterra.com. 
encaminadas a reestructurar el sector, en términos de las nuevas exigencias que se han presentado tanto en el mercado internacional de lácteos así como al interior de sus economías.

\section{Bibliografía}

The Australian Bureau of Agricultural and Resource Economics (ABARE), The Australian Dairy Industry, Impact of an open market in fluid milk supply, Report to the Federal Minister For Agriculture, Fisheries and Forestry, enero, 2001.

Australian Dairy Corporation, Annual Report, 1989-1990.

Australian Dairy Industry Council, The comprehensive reference to the Australian Dairy Industry, Morescope Publishing Pty Ltd., 1996.

Muñoz Rodríguez, Manrubio y Odermatt Pius, El sistema lecèe de México en el marco del Tratado Trilateral de Libre Comercio, Universidad Autónoma de Chapingo, noviembre de 1991.

OECD, Agricultural policies in OECD countries at a glance, 2004.

Velázquez, Víctor Manuel, Roberto García M. y Miguel Martínez D., "Análisis comparativo de los costos de producción de leche de vaca en siete países", en Políticas Agrícolas, vol. III, núm. 3, 1998.

World Bank Discussion Papers 105. Redefining the role of government in agriculture for the 1990s. Odin Knudsen, John Nash, with contributions by James Bovard, Bruce Gardner, L. Alan Winters. Washington, DC, agosto, 1993. 